\title{
Database-Based Estimation of Liver Deformation Under Pneumoperitoneum for Surgical Image-Guidance and Simulation
}

\author{
SF Johnsen ${ }^{1,2}$, S Thompson ${ }^{1}$, MJ Clarkson ${ }^{1}$, M Modat $^{1,2}$, Y Song $^{1}$, J Totz $^{1}$, \\ K Gurusamy $^{3}$, B Davidson ${ }^{3}$, ZA Taylor ${ }^{4}$, DJ Hawkes ${ }^{1}$, S Ourselin ${ }^{1,2}$ \\ 1 University College London, Centre for Medical Image Computing, London, UK \\ 2 University College London, Translational Imaging Group, CMIC, London, UK \\ ${ }^{3}$ University Dept Surgery, Royal Free Campus, UCL, London UK \\ ${ }^{4}$ University of Sheffield, CISTIB, Insigneo, Sheffield, UK
}

\begin{abstract}
The insufflation of the abdomen in laparoscopic liver surgery leads to significant deformation of the liver. The estimation of the shape and position of the liver after insufflation has many important applications, such as providing surface-based registration algorithms used in image guidance with an initial guess and realistic patient-specific surgical simulation.

Our proposed algorithm computes a deformation estimate for a patient subject from a database of known insufflation deformations, as a weighted average. The database is built from pre-operative and intra-operative 3D image segmentations. The estimation pipeline also comprises a biomechanical simulation to incorporate patient-specific boundary conditions (BCs) and eliminate any non-physical deformation arising from the computation of the deformation as a weighted average.

We have evaluated the accuracy of our intra-subject registration, used for the computation of the displacements stored in the database, and our liver deformation predictions based on segmented, in-vivo porcine CT image data from 5 animals and manually selected vascular landmarks. We found root mean squared (RMS) target registration errors (TREs) of 2.96-11.31 mm after intra-subject registration. For our estimated deformation, we found an RMS TRE of $5.82-11.47 \mathrm{~mm}$ for four of the subjects, on one outlier subject the method failed.
\end{abstract}

\section{Introduction}

Many of the algorithms currently being proposed for the registration of the pre-operative segmentation to intra-operative surface reconstructions [10] in image-guided laparoscopic liver surgery (e.g. Iterative Closest Points method) suffer from a tendency to converge to local cost-function minima when not well initialised. The main application for our pneumoperitoneum-deformation estimates is the initialisation of such registrations and thus avoid a manual initialisation step, which is desirable for both workflow and accuracy reasons. They could also help produce better patient-specific surgical simulations for the planning of a laparoscopic procedure.

Few algorithms have been proposed for this particular purpose: Work conducted by Bano et al. [1], investigated the possibility of simulating pneumoperitoneum to guide 
port placement. Their method was based on a finite element simulation of the patient abdomen which modelled the effects of the gas pressure and gravity. While it was not their primary objective, they also evaluated their method's ability to predict the position of the abdominal viscera in a pig model, and found errors in terms of mesh vertex distances of $5.7 \pm 4.9 \mathrm{~mm}$ with the larger errors being concentrated near the liver. A complete pipeline, from image segmentation through to visual output to the surgeon, for the same purpose was presented by Kitasaka et al. [5]. The tissue biomechanical model they employed was a mass-spring one to which they directly applied gas-pressure forces. However, none of the simulation parameters were given and no registration error evaluation was performed. Bosman et al. [2] looked at different ways of incorporating ligaments in surgical simulations, with pneumoperitoneum being one application. While their experiments were purely synthetic, the work does illustrate how difficult it is to generate a model that correctly takes into account all the effects of surrounding anatomy on the liver configuration under pneumoperitoneum.

Atlas-based methods have been proposed for a number of related tasks. Clements et al. [3] presented a method for the compensation of liver deformation in open hepatic surgery. Their deformation estimates were computed as a weighted average of solutions obtained with a computational model of the patient liver. Their results, while good, were obtained with only simple deformations being applied to a physical liver phantom. Plantefeve et al. [9] developed an atlas-based transfer of FEM BCs. The atlas BCs were computed with Principal Component Analysis performed on a large number of input segmentations of ligaments, blood vessels, and similar structures. Their method yielded good results, but it was limited to the mapping of easily identifiable anatomy, and no validation involving the multitude of interactions determining the liver deformation in real surgery was performed.

In this submission we propose a novel algorithm that extrapolates for a patient liver a likely deformation based on a database containing known insufflation deformations. It is more akin to the aforementioned atlas-based methods, and motivated by the assumption that accurately modelling the interactions occurring during insufflation between the liver and the surrounding tissues is too challenging, since the latter largely consist of tissues that due to their softness and mobility cannot be reliably segmented, meshed and modelled. The data required to build the database have to be acquired with dense intra-operative 3D imaging, but once the database is built it can be used at other sites where such modalities are not available.

\section{Methods}

\subsection{Algorithm Overview}

The proposed algorithm computes for a subject with an unknown liver configuration under pneumoperitoneum (unseen subject) an estimate for the displacement of its liver due to pneumoperitoneum. The estimate is computed as a weighted average of displacements observed in other subjects (training subjects) by means of intra-operative imaging and stored in a database. In the simplest case, the database only has to store for the training subjects the original pre-operative liver mesh, an augmented spectral embedding of that mesh, the displacements, and the insufflation pressure. The spectral 
embedding is used for registration with unseen-subject livers. The original liver mesh and the gas pressure are used in the computation of the weights used in the averaging process.

The algorithm comprises two processing pipelines (Fig. 1): 1) A pipeline processing training data given by $\mathrm{CT}$ images of the pre-operative and the intra-operative configuration of the abdomen, and corresponding anatomical segmentations. 2) A pipeline for the estimation of the deformation due to pneumoperitoneum for unseen subjects. This pipeline only requires a pre-operative segmentation as input.

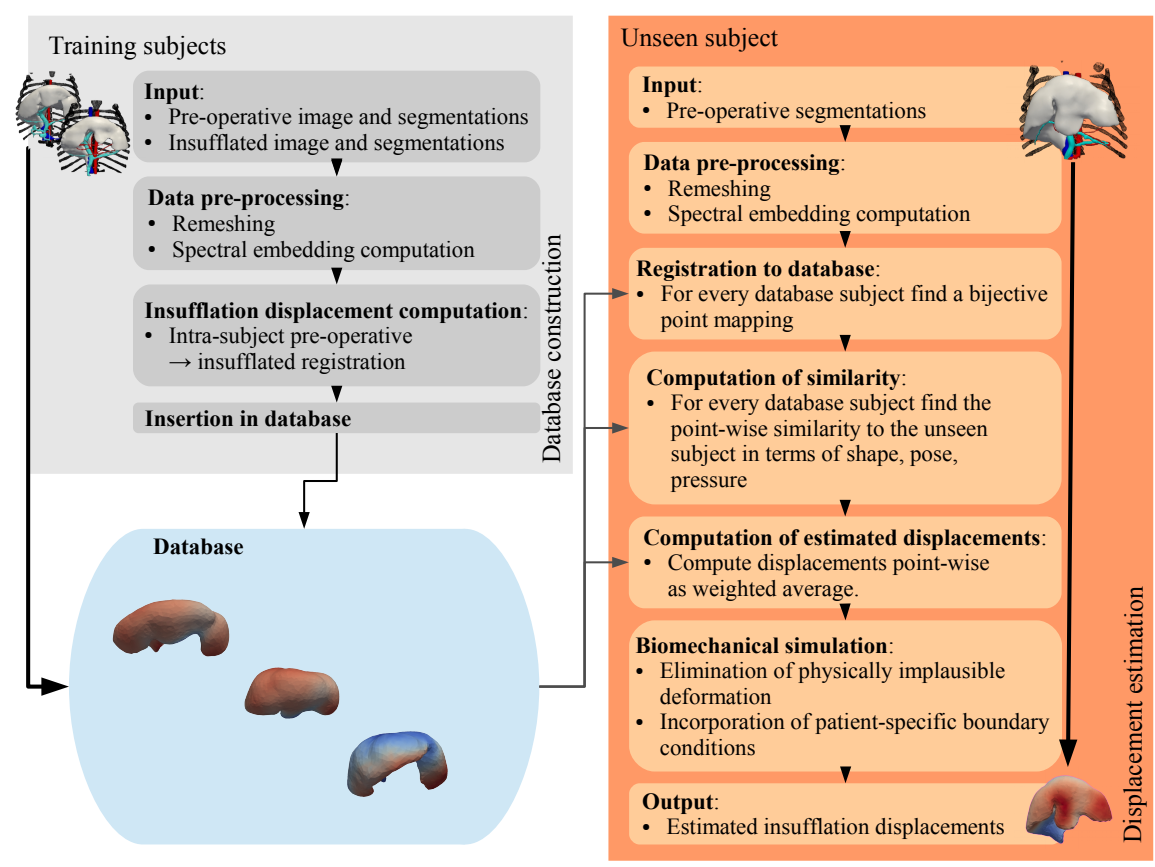

Fig. 1. Flowchart overview over the proposed deformation estimation pipeline. Left: processing of training data. Right: processing of an unseen subject for displacement estimation.

\subsection{Image Data and Segmentations}

For this work we had segmented, contrast-enhanced CT images of the pre-operative and insufflated anatomy of 5 pigs (subsequently referred to as P1, P2, P3, P4, and P5). The pigs were anaesthetised with isoflurane. They were insufflated with $\mathrm{CO}_{2}$ at a pressure of approx. $12 \mathrm{mmHg}$, in one case (P1) we assumed a pressure of $16 \mathrm{mmHg}$ due to an approx. 50\% higher mean displacement compared to other $12 \mathrm{mmHg}$ subjects, and in one case the pressure had to be lowered to $8 \mathrm{mmHg}$ (P5). A total of 4-5 ports were put in place (1 umbilical, 1 epigastric, 1 left upper quadrant, and 1 or 2 right upper quadrant ports). The CT images were acquired at full exhale, with a resolution of $0.85 \times$ 
$0.85 \times 2.5 \mathrm{~mm}$. All pigs were scanned with the same scanner (GE LightSpeed16). The segmentations were done by Visible Patient (http://www.visiblepatient.com/ en/service/), and all included the liver, the hepatic artery, the hepatic and portal vein tree, the gall bladder, and the rib cage.

\subsection{Registration}

Both the intra-subject registration, required to determine the displacements in training subjects, and the inter-subject registration of unseen subjects to the database rely on a mesh registration using spectral point correspondences.

Spectral Embeddings The spectral method establishes correspondences based on the shape topology rather than spatial configurations. This is highly advantageous for this application, since the relative size of pig liver lobes can vary considerably. The livers are also highly deformable and their lobes are very mobile. The basic form of our spectral embeddings is based on ref. [4] and is derived from bending and scale invariate geodesic distances of the different parts of the liver surface. The affinity matrix $A$, whose dominant eigenmodes form the spectral embedding, is computed as follows, with $D$ denoting the matrix of the mutual geodesic distances of the liver-mesh surface nodes:

$$
A_{i j}=\exp \left(-\alpha D_{i j}^{2}\right), \alpha=4 / \max _{k, l} D_{k l}^{2}
$$

To guide the registration algorithm, the following structures are projected onto the liver: the centreline of the trunks of the hepatic and portal veins and the hepatic artery, and the gall bladder. The projection attributes are defined on all liver-mesh nodes and consist of values inversely proportional to the node's shortest distance to the projected structure. For a projected feature mesh $Y$, they are of the following type:

$$
{ }^{Y} a\left(\boldsymbol{x}_{\boldsymbol{i}}\right)=\exp \left(-\beta\left\|\boldsymbol{x}_{i}-\arg \min _{\boldsymbol{y}_{\boldsymbol{j}} \in Y}\right\| \boldsymbol{y}_{\boldsymbol{j}}-\boldsymbol{x}_{\boldsymbol{i}}\|\|^{2}\right), \beta>0, \boldsymbol{x}_{\boldsymbol{i}} \in\{\text { Liver-mesh nodes }\}
$$

This results in an augmented spectral embedding $\hat{\boldsymbol{X}}$ consisting of the first six eigenmodes of (1) and four projection components defined by (2).

Spectral Point Correspondences Point correspondences are established by non-rigidly registering the augmented spectral embeddings [4]. The registration is done with the Coherent Point Drift (CPD) algorithm [8]. This is mathematically consistent with applications to 4+ dimensional data. For mapping attributes from one subject onto another after establishment of correspondences, a Gaussian interpolation in spectral space is employed, as done by Lombaert et al. [6].

Intra-Subject Registration To make the most use of the available data an intensitybased registration of the CT images corresponding to the segmentations used for building the database is performed with the velocity-field algorithm of NiftyReg [7]. The image registration is initialised with the result of the spectral mesh registration and augmentations are applied to the images by blending the CT images with the segmentation masks corresponding to the hepatic vein, portal vein, gall bladder, and hepatic artery. 


\subsection{Displacement Computation}

The displacement computation for an unseen subject requires a registration and known point-wise correspondences to every liver in the database. With these mutual point correspondences in place, the displacement field is computed node-wise as a weighted average of the corresponding point displacements found in the database. The following three similarity metrics are employed as weights:

Embedding similarity: $s_{S}$ is given by the similarity of the unwarped, augmented spectral embeddings of the unseen mesh $u$ and the training mesh $t$. For the purpose of the metric's evaluation the spectral embedding is treated like any multivariate node attribute that can be mapped from one subject onto another.

$$
{ }^{u t} s_{S}=-\left\|\hat{\boldsymbol{x}}\left(\boldsymbol{x}_{\boldsymbol{u}}\right)-\hat{\boldsymbol{x}}\left(\phi_{\boldsymbol{u} 2 \boldsymbol{t}}\left(\boldsymbol{x}_{\boldsymbol{u}}\right)\right)\right\| /\left\|\hat{\boldsymbol{x}}\left(\boldsymbol{x}_{\boldsymbol{u}}\right)\right\|
$$

where $\phi_{\boldsymbol{u} \boldsymbol{2} t}\left(\boldsymbol{x}_{\boldsymbol{u}}\right)$ is the coordinate mapping from $u$ to $t$, and thus $\hat{\boldsymbol{x}}\left(\phi_{\boldsymbol{u} \boldsymbol{2} t}\left(\boldsymbol{x}_{\boldsymbol{u}}\right)\right)$ the spectral embedding value of the point on $t$ corresponding to $\boldsymbol{x}_{\boldsymbol{u}}$.

Transform similarity: $s_{X}$ is computed from local $3 \times 3$ affine transforms $P_{t 2 u}$, in turn computed from the spectral point correspondences, by penalising departures from the identity transform (I):

$$
{ }^{u t} s_{X}=-\sum_{i, j}\left|\left(P_{t 2 u}\right)_{i j}-\mathrm{I}_{i j}\right|
$$

Gas-pressure similarity: $s_{G}$ is computed from the relative difference in insufflation gas pressure $(p)$ :

$$
{ }^{u t} s_{G}=-\left|p_{u}-p_{t}\right| / p_{u}
$$

The computation of the similarity for all nodes of the unseen mesh and all training meshes results in three $N \times M$ similarity matrices $S$, where $N$ is the number of nodes and $M$ is the number of training subjects. These are then normalised via

$$
\tilde{S}=(S-\min S) /(\max S-\min S)
$$

Finally, the predicted displacements $\boldsymbol{u}_{\boldsymbol{p}}$ are computed at every node of the output mesh from the following weighted average:

$$
\boldsymbol{u}\left(\boldsymbol{x}_{\boldsymbol{u}}\right)=\frac{1}{\sum_{\tau \in\{\text { database }\}}{ }^{u \tau} \tilde{s}_{S}{ }^{u \tau} \tilde{s}_{X}{ }^{u \tau} \tilde{s}_{G}} \sum_{t \in\{\text { database }\}}{ }^{u t} \tilde{s}_{S}{ }^{u t} \tilde{s}_{X}{ }^{u t} \tilde{s}_{G} \boldsymbol{u}\left(\phi_{u \mathbf{z} t}\left(\boldsymbol{x}_{\boldsymbol{u}}\right)\right) \frac{p_{u}}{p_{t}}
$$

The factor $p_{u} / p_{t}$ provides a first-order approximation for the displacement magnitude as a function of pressure.

After a first displacement field is computed with (7) one has the option to impose this surface displacement field as a boundary condition on a biomechanical simulation to obtain a displacement solution for the liver parenchyma. If the boundary conditions are formulated as penalty forces one further has the means to eliminate any physically implausible deformation from the solution, e.g. excessive volume change. This solution can also easily be combined with patient-specific boundary conditions extracted from the patient CT, such as zero-displacement constraints at attachment points of major vasculature. The settings of these simulations can be optimised on the training data.

If the database is large, by introducing a reference liver geometry to which the unseen liver and all training livers are registered, one can evaluate (7) with one intersubject registration per unseen subject. 


\section{Experiments}

(a) Intra-subject registration errors (RMS TRE):

\begin{tabular}{|l|c|c|c|c|c|}
\hline & P1 & P2 & P3 & P4 & P5 \\
\hline Rigid alignment & 13.83 & 13.61 & 16.27 & 12.53 & 9.69 \\
\hline Mesh registration & 8.00 & 7.65 & 11.31 & 9.54 & 4.29 \\
\hline $\begin{array}{l}\text { Mesh + image reg- } \\
\text { istration }\end{array}$ & 3.88 & 3.52 & 12.04 & 3.80 & 2.96 \\
\hline $\begin{array}{l}\text { Surface displacement } \\
\text { (max/mean/min) }\end{array}$ & $65.8 / 41.4 / 6.3$ & $51.6 / 19.4 / 1.34$ & $33.4 / 15.1 / 0.2$ & $57.2 / 19 / 1.3$ & $28.4 / 13.6 / 1.0$ \\
\hline
\end{tabular}

(b) RMS TREs arising from application of the displacement predicted with (7)

\begin{tabular}{|c||c|c|}
\hline Subject & Weighted-average displacement & $\begin{array}{l}\text { Weighted-average displacement and } \\
\text { biomechanical simulation }\end{array}$ \\
\hline P1 & 37.57 & 34.98 \\
\hline P2 & 7.73 & 8.89 \\
\hline P3 & 13.89 & 11.47 \\
\hline P4 & 8.61 & 10.53 \\
\hline P5 & 6.03 & 5.82 \\
\hline
\end{tabular}

Table 1. RMS registration errors. All values in $\mathrm{mm}$.

\subsection{Intra-Subject Registration}

The validation of the intra-subject registration pipeline is based on easily identifiable vascular landmarks evenly distributed throughout the liver parenchyma. Per subject we used 6-8 landmarks. The RMS TREs are listed in Table 1(a). The first row shows the RMS errors after an alignment of the centre of gravity of the segmentation masks. The last row of Table 1(a) shows the max, mean, and min surface displacement computed with the full pipeline. An example of the images produced in the various stages of the pipeline can be seen in Fig. 2(a).

The TREs in Table 1(a) indicate that the surface registration handles large deformations well - this is especially evident in the case of P1, but to achieve the best possible registration error in most cases an intensity-based image registration step is required. In all cases but P3, whose images are severely degraded by breathing motion artefacts, the RMS error obtained after the image registration is below $5 \mathrm{~mm}$.

\subsection{Displacement Estimation}

Table 1(b) lists the RMS TREs obtained with a leave-one-out analysis of the predicted displacements on the same landmarks as used for validation of the intra-subject registration pipeline. The first data column in Table 1(b) contains the RMS TREs obtained 


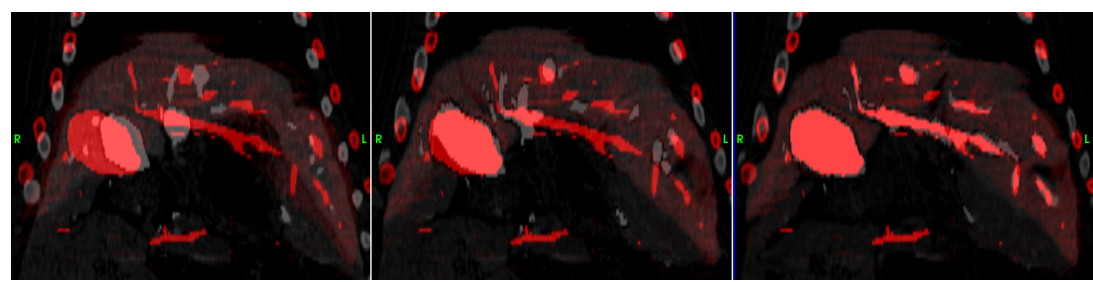

(a) The CT images corresponding to the different stages of the intra-subject registration pipeline with target image overlaid (red): coronal slices through images of P2; Left to right: enhanced source image; surface-registration result; result of intensity-based registration.
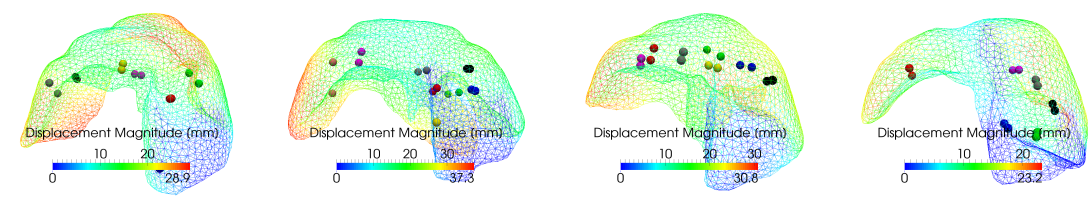

(b) Displacement-estimation pipeline final results with landmarks (left to right): P2, P3, P4, P5; displacement magnitude colour mapped; corresponding landmarks (source, target) shown as spheres with same colour.

Fig. 2. Result configurations of the intra-subject registration pipeline and the displacement estimation pipeline

with the weighted-average database displacements given by (7); the second column lists the errors obtained when additionally running a biomechanical simulation as described towards the end of Sect. 2.4.

The full pipeline results in Table 1(b) show: on all subjects except P1 the RMS TRE is comparable to the registration error after the intra-subject surface registration and significantly better than what is obtained with a rigid alignment of the pre- and postinsufflation meshes (Table 1(a)). P1 is an outlier not only in terms of its displacement magnitude but also due to segmentation errors leading to lobes being fused together in two places and the liver being slightly rotated about the ventro-dorsal axis. Since there is no match in the database for such a liver, the method can only fail. The biomechanical simulation seems to be most beneficial where the weighted average displacement computed with (7) works poorly, in particular on subject P1.

\section{Conclusions}

We have presented a new method that can approximate the effects on the liver of the complex mechanical interactions of the abdominal viscera occurring during gas insufflation in laparoscopic surgery. The displacements are computed node-wise as the weighted average of displacements observed in training subjects where the insufflated configuration was captured with CT imaging. The weights are provided by custom similarity metrics comparing unseen and training data. 
The proposed method achieves an error that is comparable to what has been reported for methods relying on sophisticated biomechanical modelling with a $5.82-11.47 \mathrm{~mm}$ RMS TRE on subsurface landmarks. While it has some disadvantages handling outliers, it achieves so without using any intra-operatively acquired data for the unseen subject whatsoever and with a very small database of training subjects. Further, considering that the biomechanical simulation currently only acts as a rudimentary safeguard against non-physical deformation and the method can be easily combined with more advanced biomechanical modelling, the current implementation likely only hints at its true potential. This is also true when considering applications to human livers where the inter-patient variability is not as pronounced as in pigs and consistent segmentation is simpler. It was also shown that the intra-subject registration component when used on its own can provide sub-surface accuracy in the range 3-4mm, which is more than adequate for the intended application.

Acknowledgements The authors were supported by the following funding bodies and grants: EPSRC EP/H046410/1, NIHR BRC UCLH/UCL High Impact Initiative BW.mn.BRC10269, UCL Leonard Wolfson Experimental Neurology Centre, EU-FP7 FP7-ICT-2011-9-601055. The data was supplied by the Wellcome/DoH HICF project T4-317.

\section{References}

1. J Bano, A Hostettler, S A Nicolau, C Doignon, and H S Wu. Simulation of the Abdominal Wall and Its Arteries after Pneumoperitoneum for Guidance of Port Positioning in Laparoscopic Surgery. In 8th International Symposium, ISVC 2012, pages 1-11, 2012.

2. J Bosman, N Haouchine, J Dequidt, and I Peterlik. The Role of Ligaments: Patient-Specific or Scenario-Specific? In Fernando Bello and Stéphane Cotin, editors, 6th International Symposium, ISBMS 2014, volume 8789 of LNCS, pages 228-232, 2014.

3. L W Clements, P Dumpuri, W C Chapman, R L Galloway, and M I Miga. Atlas-based method for model updating in image-guided liver surgery. In Proceedings of SPIE, volume 6509, pages 650917-650917-12. SPIE, 2007.

4. V Jain, H Zhang, and O Van Kaick. Non-rigid spectral correspondence of triangle meshes. International Journal of Shape Modeling, 13(01):101-124, June 2007.

5. T Kitasaka, K Mori, and Y Hayashi. Virtual pneumoperitoneum for generating virtual laparoscopic views based on volumetric deformation. Medical Image Computing and ComputerAssisted Interventation - MICCAI 2004, 3:559-567, 2004.

6. H Lombaert, J Sporring, and K Siddiqi. Diffeomorphic Spectral Matching of Cortical Surfaces. Information Processing in Medical Imaging, pages 376-389, 2013.

7. M Modat, P Daga, M J Cardoso, S Ourselin, G R Ridgway, and J Ashburner. Parametric non-rigid registration using a stationary velocity field. In Proceedings of the Workshop on Mathematical Methods in Biomedical Image Analysis, pages 145-150, 2012.

8. A Myronenko and X Song. Point set registration: coherent point drift. IEEE transactions on pattern analysis and machine intelligence, 32(12):2262-75, December 2010.

9. R Plantefeve, I Peterlik, H Courtecuisse, R Trivisonne, J-P Radoux, and S Cotin. Atlas-based transfer of boundary conditions for biomechanical simulation. In Medical Image Computing and Computer-Assisted Interventation - MICCAI 2014, volume 8674 of LNCS, pages 33-40, 2014.

10. J Totz, S Thompson, D Stoyanov, K Gurusamy, B R Davidson, D J Hawkes, and M J Clarkson. Fast Semi-dense Surface Reconstruction from Stereoscopic Video in Laparoscopic Surgery. Information Processing in Computer-Assisted Interventions - IPCAI 2014, pages 206-215, 2014. 